\title{
Crystal structure of tripraseodymium hexasiliconundecanitride, $\operatorname{Pr}_{3} \mathrm{Si}_{6} \mathbf{N}_{11}$
}

\section{T. Schlieper and W. Schnick}

Universität Bayreuth, Laboratorium für Anorganische Chemie, D-95440 Bayreuth, Germany

Received May 16, 1995, transferred to database ICSD March 1996, CSD-No. 402178

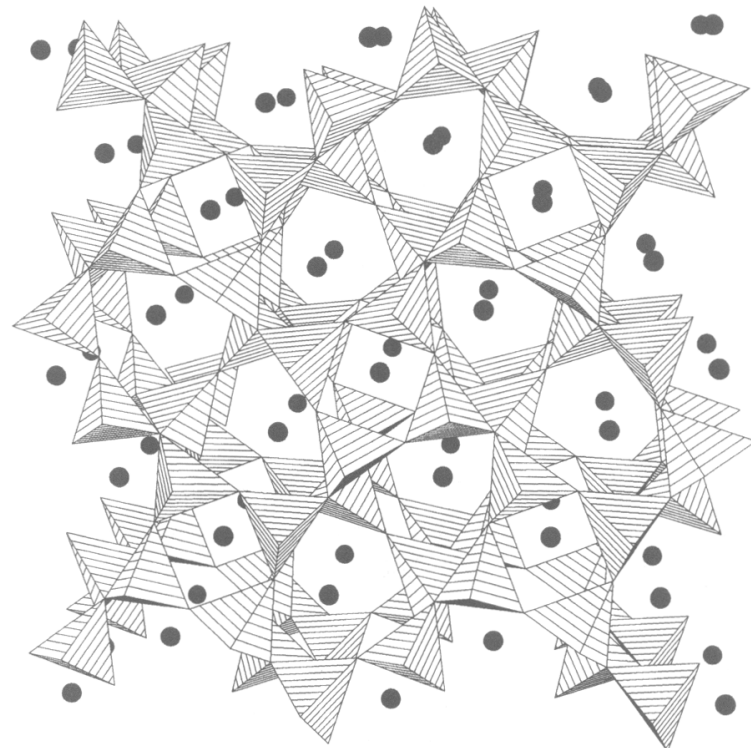

Source of material: $\operatorname{Pr}_{3} \mathrm{Si}_{6} \mathrm{~N}_{11}$ was synthesized by reaction of elemental praseodymium with silicon diimide under nitrogen atmosphere at $1848 \mathrm{~K}$ in a high frequency furnace.

The pure compound was obtained as transparent greenish crystals. The single crystal structure determination of an isotypic compound $\left(\mathrm{Ce}_{3} \mathrm{Si}_{6} \mathrm{~N}_{11}\right)$ is described earlier (see ref. 1). $\mathrm{Pr}_{3} \mathrm{Si}_{6} \mathrm{~N}_{11}$ contains $\operatorname{Pr}^{3+}$ as well as a polar three-dimensional covalent network of corner sharing $\mathrm{SiN}_{4}$ tetrahedra.
Table 1. Parameters used for the X-ray data collection

\begin{tabular}{ll}
\hline & \\
Crystal: & greenish prisma, size $0.2 \times 0.15 \times 0.1 \mathrm{~mm}$ \\
Wavelength: & Mo $K_{\alpha}$ radiation $(0.71073 \AA)$ \\
$\mu:$ & $152.94 \mathrm{~cm}^{-1}$ \\
Diffractometer: & Siemens P4 \\
Scan mode: & $\omega$ \\
$\mathrm{T}_{\text {measurement: }}$ & $296 \mathrm{~K}$ \\
$2 \theta_{\text {max }}:$ & $60^{\circ}$ \\
$\mathrm{N}(h k l)_{\text {unique }}:$ & 774 \\
Criterion for $F_{\mathrm{o}}:$ & $F_{0}>0 \sigma\left(F_{\mathrm{o}}\right)$ \\
$\mathrm{N}(\text { param })_{\text {refined }}:$ & 54 \\
Program: & SHELXTL-plus
\end{tabular}

$\mathrm{N}_{11} \mathrm{Pr}_{3} \mathrm{Si}_{6}$, tetragonal, $P 4 b m$ (No. 100), $a=10.099(1) \AA$,

$c=4.843(1) \AA, V=493.9 \AA^{3}, Z=2, R(F)=0.026, R_{\mathrm{w}}(F)=0.026$.

Table 2. Final atomic coordinates and displacement parameters (in $\AA^{2}$ )

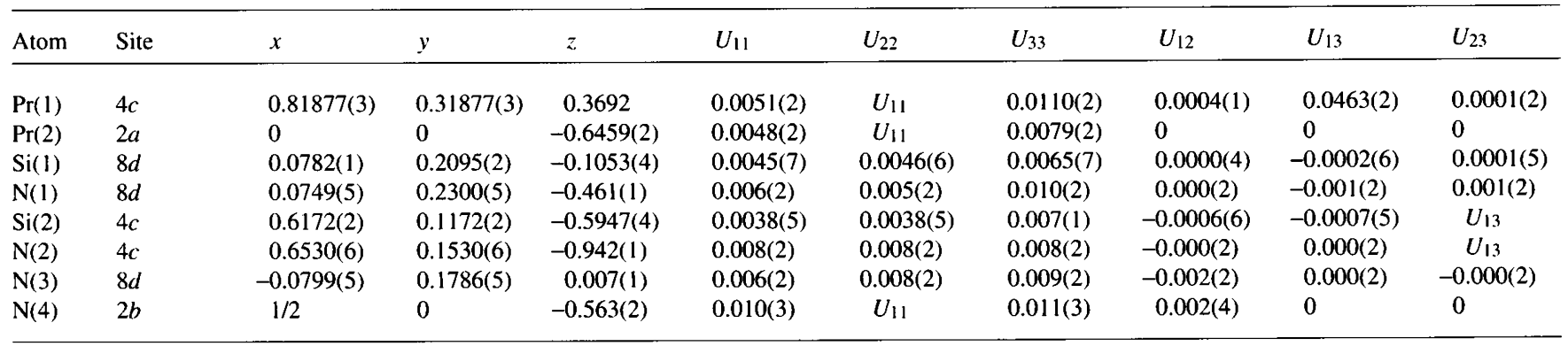

\section{Reference}

1. Schlieper, T.; Schnick, W.: Synthese, Kristallstruktur und magnetische Eigenschaften von $\mathrm{Ce}_{3} \mathrm{Si}_{6} \mathrm{~N}_{11}$. Z. anorg. allg. Chem. 621 (1995) 1535. 